\title{
Locus-Specific Genomic DNA Purification Using the CRISPR System: Methods and Applications
}

\author{
Hirotaka Fujita, Toshitsugu Fujita, and Hodaka Fujii ${ }^{*, \dagger}$
}

\begin{abstract}
A multitude of molecular interactions with chromatin governs various chromosomal functions in cells. Insights into the molecular compositions at specific genomic regions are pivotal to deepen our understanding of regulatory mechanisms and the pathogenesis of disorders caused by the abnormal regulation of genes. The locusspecific purification of genomic DNA using the clustered regularly interspaced short palindromic repeats (CRISPR) system enables the isolation of target genomic regions for identification of bound interacting molecules. This CRISPR-based DNA purification method has many applications. In this study, we present an overview of the CRISPR-based DNA purification methodologies as well as recent applications.
\end{abstract}

\section{Introduction}

In the field of chromosome biology, it is an enigma how genomic events are regulated so precisely. A large number of molecular interactions occur with chromatin to modulate gene transcription, DNA replication or repair, and other critical genomic processes. ${ }^{1-3}$ Identification of these interacting components can advance the understanding of the molecular mechanisms underlying these genomic events.

The most comprehensive and unbiased approach to achieve this goal is biochemical isolation of a specific genome region while retaining the molecular interactions. Several methods have been developed for this purpose. A locus of interest can be isolated by insertion of recognition sequences of DNA-binding molecules, expression of the DNA-binding molecules in the cells, fragmentation of chromatin by sonication or other methods, followed by affinity purification of the DNA-binding molecules bound to the target locus. ${ }^{4-14}$ Cross-linking can be performed, if necessary. The locus tagged with the recognition sequences of the DNA-binding molecules can also be isolated by cross-linking, if necessary, fragmentation, and incubation with recombinant or synthetic DNA- binding molecules before affinity purification of the DNA-binding molecules bound to the target locus. ${ }^{15}$ However, this approach is tedious and requires the production of cells that retain the recognition sequences of exogenous DNA-binding molecules.

To avoid this tedious step, the proteomics of isolated chromatin (PICh) assay, employing locked nucleic acids containing biotinylated oligonucleotides, can be used to isolate telomeres or centromeres for identification of their associated proteins. ${ }^{16-18}$ Previously, PICh was restricted to genomic regions containing multiple repeats or abundant targets due to a low isolation efficiency. Recent advancements enable the isolation of a more complex target of ribosomal DNAs. ${ }^{19}$ However, PICh requires the reoptimization of probe sequences and purification conditions, thereby limiting its adaptability to different genomic targets. Thus, more flexible and efficient techniques are necessary for the purification of target loci.

The advancement of engineered DNA-binding molecules has made it easier to purify a locus of interest. The zinc finger proteins and transcription activatorlike (TAL) proteins are pioneering examples of engineered DNA-binding molecules. ${ }^{20-23}$ More recently, the 
clustered regularly interspaced short palindromic repeats (CRISPR) system was adapted as a gene editing tool enabling the study of reverse genetics. ${ }^{24,25}$ Moreover, the development of nuclease-dead Cas9 (dCas9) has expanded the application of the CRISPR system beyond gene editing. ${ }^{26}$ Since dCas9 retains its ability to bind to target sequences, the catalytically inactive CRISPRdCas9 system is a programmable DNA-binding tool. ${ }^{27,28}$ The first report of a locus-specific CRISPRbased DNA purification method for subsequent identification of interacting molecules was published in 2013. ${ }^{29}$ Since then, about 30 articles using the method have already been published (summarized in Fig. 1). The CRISPR-based DNA purification system utilizes the catalytically inactive CRISPR complex to form a tagging complex for isolation of the target locus. Interacting molecules bound to the locus of interest are identified first by isolation of the target locus by affinity purification, followed by subsequent identification of binding molecules. Thus, this is a powerful technique to purify the target locus while retaining the interaction between DNA and its binding components, which provides comprehensive insights into the interacting molecules by use of downstream biochemical applications.

\begin{tabular}{|c|c|c|c|c|c|}
\hline Year & Epitope tags & Tagging methods & $\begin{array}{l}\text { Downstream } \\
\text { applications }\end{array}$ & Organisms and/or cell types & References \\
\hline 2013 & 3xFLAG & in-cell & MS & $293 T$ & Fujita and Fujii ${ }^{29}$ \\
\hline \multirow[t]{2}{*}{2014} & 3xFLAG & in-cell & MS (SILAC) & HT1080 & Fujita and Fujii 55 \\
\hline & Protein A & in-cell & MS & S. cerevisiae & Waldrip et al. ${ }^{48}$ \\
\hline \multirow[t]{2}{*}{2016} & 3xFLAG, biotin & in vitro & PCR & $293 T$ & Fujita et al. ${ }^{30}$ \\
\hline & $3 x F L A G$ & in-cell and in vitro & PCR & HCT116 & Fujita et al. ${ }^{83}$ \\
\hline \multirow[t]{4}{*}{2017} & (dCas9) & in-cell & RNA-Seq & ASPC-1 & Zhang et al. ${ }^{65}$ \\
\hline & $3 \times F L A G$ & in-cell & NGS & K562 & Fujita et al. 68 \\
\hline & biotin & in-cell & MS (iTRAQ) and $3 C$ & K562 & Liu et al. ${ }^{42}$ \\
\hline & $3 \times F L A G$ & in vitro & NGS & DT40 & Fujita et al. ${ }^{12}$ \\
\hline \multirow[t]{9}{*}{2018} & 3xFLAG, 2xAM & in-cell & PCR & $293 T$ & Fujita et al. ${ }^{82}$ \\
\hline & FLAG & in-cell & MS & TNBC & Han et al. 51 \\
\hline & $3 \times F L A G$ & in-cell & MS & MB135 & Campbell et al. ${ }^{52}$ \\
\hline & 3xFLAG & in vitro & MS & $\mathrm{S} 2$ and $\mathrm{HeLa}$ & Tsui et al. ${ }^{46}$ \\
\hline & $3 \times F L A G$ & in-cell & PCR & $\begin{array}{l}\text { primary CD4 (+) T cells from } \\
\text { 3xFLAG-dCas9 Tg mouse }\end{array}$ & Fujita et al. ${ }^{84}$ \\
\hline & 3xFLAG & in-cell & MS (SILAC) & SK-N-BE(2)c & Hamidian et al. 56 \\
\hline & $3 \times F L A G$ & in-cell & PCR & E. coli & Fujita et al. ${ }^{85}$ \\
\hline & $\mathrm{HA}$ & in-cell & MS and NGS & primary chondrocytes & Mochizuki et al. ${ }^{49}$ \\
\hline & (dCas9) & in-cell & RNA-Seq & MDA-MB-231 & Chen et al. ${ }^{66}$ \\
\hline \multirow[t]{4}{*}{2019} & $\mathrm{HA}$ & in-cell & PCR & $293 T$ & Kuscu et al. ${ }^{86}$ \\
\hline & biotin & in vitro & NGS & E. coli & Lee et al. ${ }^{39}$ \\
\hline & biotin & in vitro & NGS & $\mathrm{CHO}$ & Slesarev et al. 40 \\
\hline & $\mathrm{HA}$ & in-cell & NGS & P. falciparum & Baumgarten et al. ${ }^{87}$ \\
\hline \multirow[t]{7}{*}{2020} & $2 \times A M$ & in-cell & PCR & $\mathrm{N} 2 \mathrm{a}$ & Carpenter et al. ${ }^{88}$ \\
\hline & $3 \times F L A G$ & in-cell & MS & NTERA & González-Rico et al. ${ }^{53}$ \\
\hline & $3 \times F L A G$ & in-cell & MS & immortalized preadipocytes & Shamsi et al. ${ }^{47}$ \\
\hline & $3 \times F L A G$ & in-cell & NGS & $2 \mathrm{~B} 4$ & Seo et al. ${ }^{71}$ \\
\hline & $3 \times F L A G$ & in-cell & MS & SW480, SW620 & Huang et al. ${ }^{54}$ \\
\hline & $3 \times F L A G$ & in-cell & IB & S. cerevisiae & Kuhl et al. ${ }^{89}$ \\
\hline & $3 \times \mathrm{HA}$ & in-cell & MS & P. falciparum & Bryant et al. 90 \\
\hline
\end{tabular}

FIG. 1. Publications using CRISPR-based purification of specific DNA species. Tags for affinity purification used in two or more publications are shown in different colors. In vitro tagging is highlighted in red. In the "Downstream applications" column, MS, RNA-Seq, and NGS are shown in red, blue, and green, respectively. All studies employed Sp-dCas9. One study used Sa-dCas9 in addition to Sp-dCas9. ${ }^{82}$ Publications are listed in order of publication date. $\mathrm{IB}$, immunoblot analysis. Color images are available online. 
In this study, we aim to outline the recent advances of the locus-specific CRISPR purification method and its applications. This review will first introduce the strategies for affinity purification of a specific genomic locus. Subsequently, we describe the downstream applications to identify the interacting molecules and compare this method with others.

\section{The Principles of Locus-Specific CRISPR-Based Purification}

The CRISPR-based locus-specific DNA purification is based on the DNA-binding ability of catalytically inactive CRISPR and chromatin immunoprecipitation (ChIP) with or without the use of antibodies (Abs). The target DNA region can be tagged with the CRISPR complex inside of the cell or in vitro. ${ }^{29,30}$

In-cell tagging

The in-cell tagging approach labels a target locus with the CRISPR complex in the cell of interest by expression of the CRISPR complex (Fig. 2). If necessary, the chromatinCRISPR complex can be cross-linked with formaldehyde or other cross-linkers to preserve the chromatin structure during purification. After chromatin fractionation, the chromatin DNA is fragmented by sonication or enzymatic digestion. In either case, shearing conditions of chromatin DNA should be optimized so that average length of chromatin DNA is between 1 and $2 \mathrm{kbp}$. The chromatin-CRISPR complex is affinity purified with antibodies (Abs) as well as other molecules recognizing either the tags fused to dCas 9 or guide RNA (gRNA), or the anti-Cas9 Ab. If applicable, the cross-linking is reversed, and either mass spectrometry (MS) is performed for associated proteins, or next-generation sequencing (NGS) or microarray analysis for RNAs and DNAs. The detailed technical variations, considerations, and downstream applications are discussed as follows.

The CRISPR machinery can be expressed transiently or stably. The transient expression of the CRISPR complex from transfected DNA or RNA, or transduction of CRISPR ribonucleoprotein (RNP) complexes is convenient for easy-to-transfect or easy-to-transduce cells. However, some cell lineages have poor expression levels or low transfection/transduction efficiency resulting in low yields of affinity purification. In such cases, the cells stably expressing the CRISPR complex should be generated using a retrovirus-, a lentivirus-, or an adeno-associated virus-derived expression system. Nowadays, researchers can easily obtain plasmids for expression in bacteria, budding yeast, and mammalian cells, including retroviral/lentiviral expression vectors for CRISPR-based DNA purification from Addgene.*

*www.addgene.org/crispr/purify
In addition, transgenic mouse lines carrying dCas 9 are options to purify the target locus from primary cells and tissues by expression of a gRNA(s) (RIKEN BioResource Center; No. RBRC09976, 10188-90).

\section{In vitro tagging}

The in vitro tagging approach captures the fragmented chromatin using a CRISPR-dCas9 complex, which is prepared in a test tube by mixing a synthesized gRNA and a recombinant dCas9 protein (Fig. 3). The advantage of in vitro tagging is that it does not require the expression of the CRISPR complex in the cell of interest. This is beneficial for the analysis of CRISPR-based DNA purification for several reasons (Fig. 4). First, the direct tagging of chromatin without the need to produce cells expressing the CRISPR complex makes it more time efficient and cost-effective. This is important in situations where expression of the CRISPR complex is difficult or impossible, such as in clinical specimens, pathogens, or specific lineages of primary cells and tissues. In addition to nuclear DNA, organelle DNA, including mitochondrial DNA (mtDNA) and chloroplast DNA, is a potential target of in vitro tagging approach. Because of the insufficient delivery of gRNA into organelles, in-cell tagging and purification of a specific region of intracellular mtDNA by the CRISPR system might be difficult. ${ }^{31,32}$ In this regard, the in vitro tagging could be a practical approach for the isolation of a region of organelle DNA, as it does not require the intracellular delivery of the CRISPR complex. Second, in vitro tagging avoids inherent risks of in-cell tagging, such as interfering with the genomic functions or altering chromatin accessibility. ${ }^{33,34}$ Moreover, the presence of the CRISPR complex at the locus might hinder the interaction of endogenous DNA-binding factors. The in vitro capture of the target locus after the fixation of chromatin eliminates these risks of in-cell tagging. However, compared with in-cell tagging, the in vitro method has a lower efficiency of affinity purification because the CRISPR complex needs to access the target locus, especially after fixation, whereby the chromatin is crosslinked. Thus, more cells might be required for identification of interacting molecules than with the in-cell tagging method.

The in vitro tagging approach can also be used for enrichment of specific DNA species from a heterogenous population of purified DNA molecules, such as fragmented whole genomic and complementary DNAs. Sequencing the enriched species of interest is more time and cost-effective than a nonselective analysis method, such as whole-genome sequencing. Various target-enrichment methods have been widely used, such 


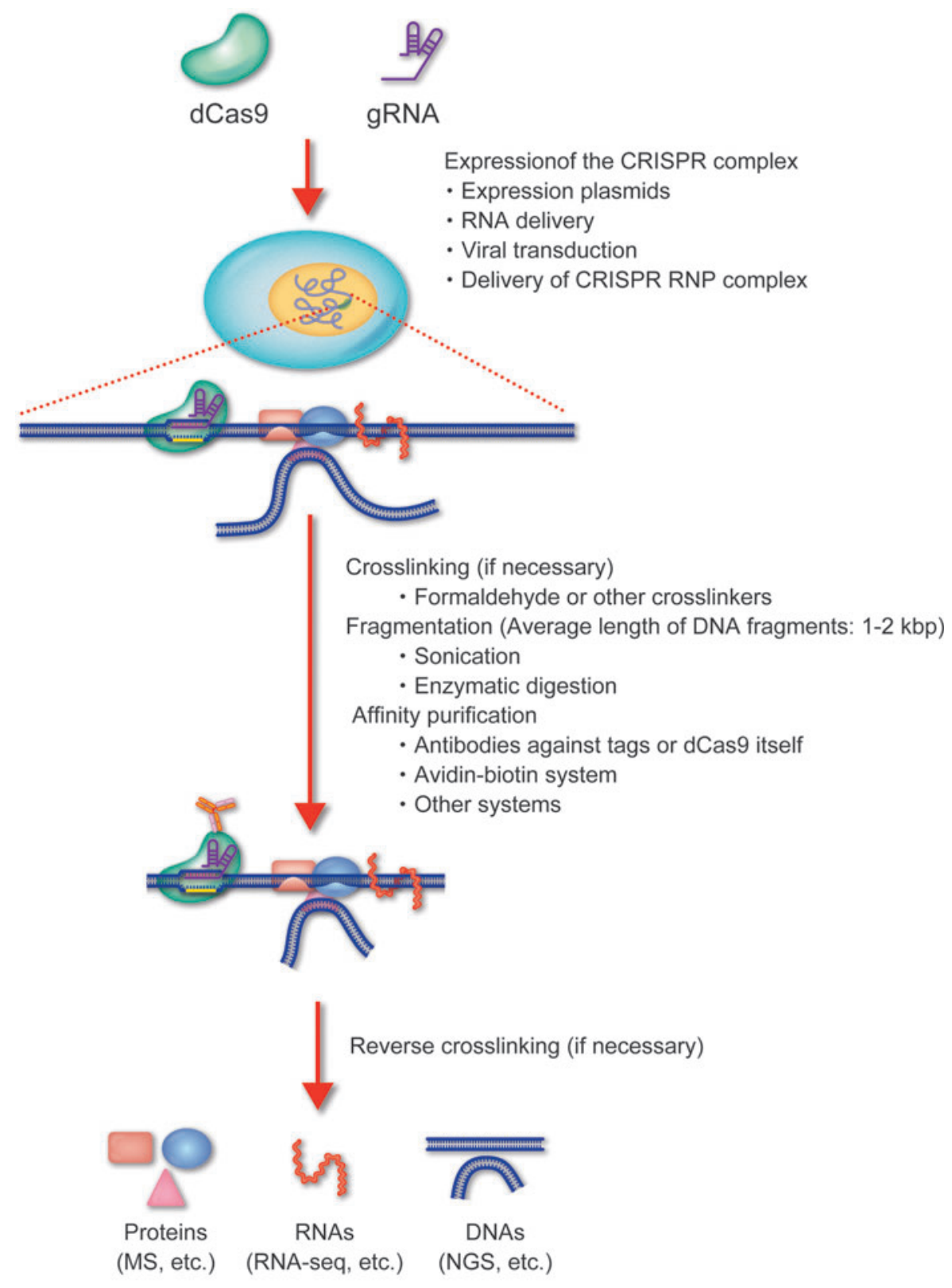

Identification of molecules associated with the target genomic region

FIG. 2. In-cell tagging of CRISPR-based DNA purification of a specific genomic region. Schematic of the in-cell tagging approach. A target locus is tagged in a cell with the CRISPR complex, and the chromatin-CRISPR complex is affinity purified. The molecules associated with the purified genomic region are identified by downstream analyses. Color images are available online.

as multiplexed polymerase chain reaction (PCR), hybridization capture using complementary strands, and selective circularization using molecular inversion probes. ${ }^{35-38}$ The combination of CRISPR-based DNA enrichment and NGS can be used to detect the rare variants and to quantify the gene copy number. ${ }^{30,39,40}$ Because the CRISPR-based DNA-enrichment approach does not require a PCR amplification step before se- quencing, this is one of the few enrichment techniques applicable to long-read sequencing. Long-read sequencing is a state-of-the-art technology for improved mapping accuracy, throughput, and identification of structural variants. ${ }^{41}$ The combination of CRISPR-based DNA purification with long-read sequencing is, therefore, not only useful for biomedical research, but also for clinical diagnostics in the future. 


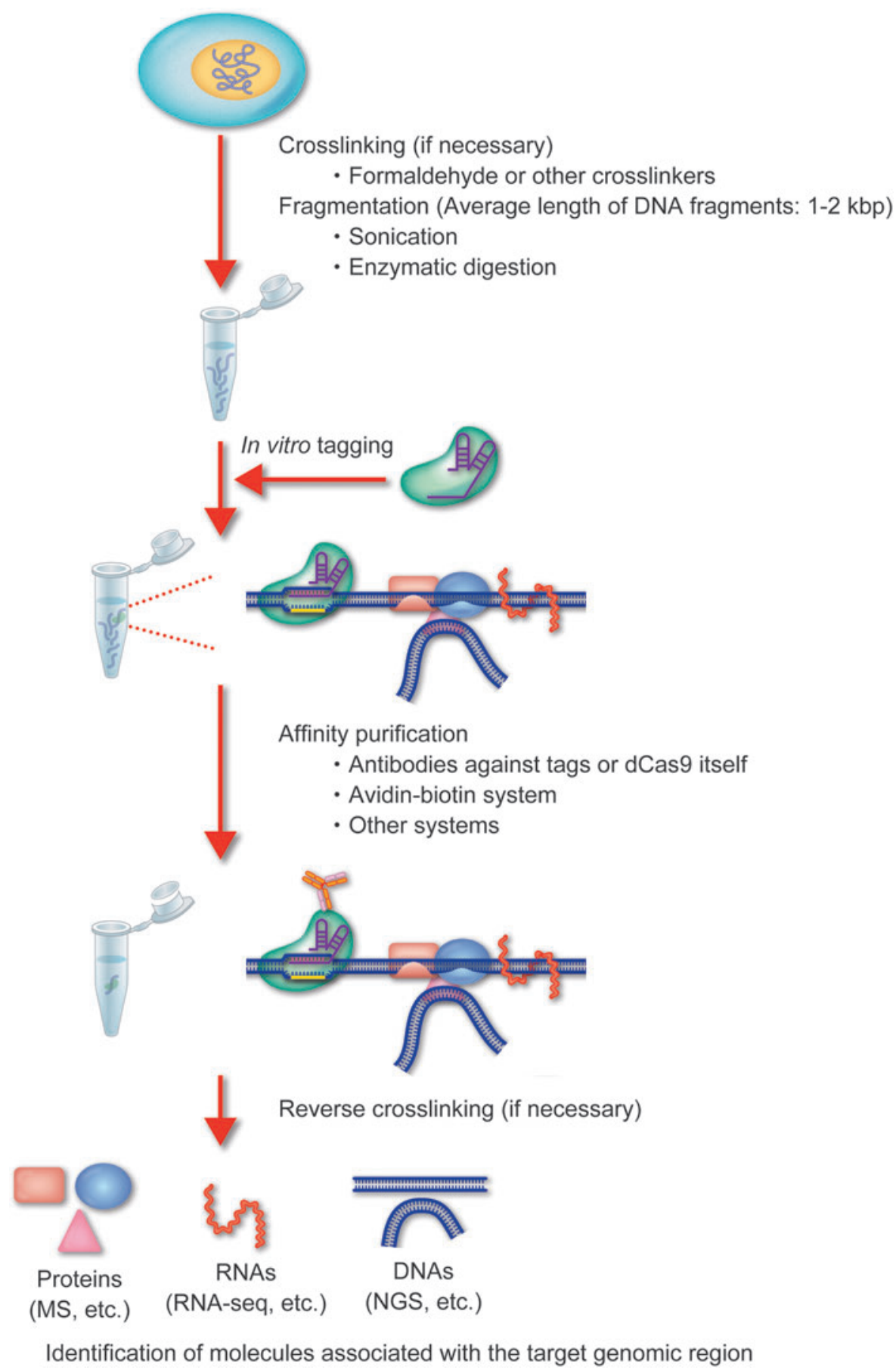

FIG. 3. In vitro tagging of CRISPR-based DNA purification of a specific genomic region. Schematic of the in vitro tagging approach. A target DNA is captured by the CRISPR complex consisting of recombinant dCas 9 and synthetic gRNA, lysed, DNA fragmented, and then purified. Color images are available online.

Tag systems of CRISPR complex

for affinity purification

CRISPR-based DNA purification of a specific locus is compatible with virtually any affinity purification systems by tagging of the CRISPR complex with $3 \times$ FLAG, pro- tein A, biotin, $2 \times \mathrm{AM}$, HA $(1 \times$ and $3 \times)$ as well as using endogenous epitopes of the CRISPR complex (Fig. 1). It has been reported that the biotin tag system using biotinylation enzymes expressed in the target cells improves the specificity and efficiency of CRISPR-based 


\begin{tabular}{|c|c|c|}
\hline & In-cell tagging & In vitro tagging \\
\hline Advantages & - Higher yields & $\begin{array}{l}\text { - No need for expression of the } \\
\text { CRISPR complex } \\
\text { - Retention of intrinsic chromatin } \\
\text { state }\end{array}$ \\
\hline Disadvantages & $\begin{array}{l}\text { - Exprssion of the CRISPR complex } \\
\text { required } \\
\text { - Potential risk to alter intrinsic } \\
\text { chromatin accessibility and function }\end{array}$ & - Lower yields \\
\hline \multirow{4}{*}{ Applications } & \multicolumn{2}{|c|}{$\begin{array}{c}\text { Nuclear DNAs of live cells } \\
\text { (cell lines, primary cells, tissues, etc.) }\end{array}$} \\
\hline & & $\begin{array}{c}\text { Fixed samples } \\
\text { (clinical specimens, pathogens, etc.) }\end{array}$ \\
\hline & & $\begin{array}{c}\text { Organelle DNAs } \\
\text { (mtDNA, chloroplast DNA) }\end{array}$ \\
\hline & & Purified DNAs \\
\hline
\end{tabular}

FIG. 4. Comparison of in-cell and in vitro tagging systems. Summary of the advantages/disadvantages and application examples of in-cell and in vitro tagging methods. Color images are available online.

DNA purification compared with the $1 \times$ FLAG tag and anti-Cas9 $\mathrm{Ab}^{42}$ However, this needs to be investigated in comparison with other high-affinity tag systems, such as $3 \times$ FLAG. Indeed, the $3 \times$ FLAG tag was more specific than the biotin tag with the in vitro tagging system. ${ }^{30}$ Because the competitive elution of $3 \times$ FLAG fusion proteins using $3 \times$ FLAG peptides is milder than the dissociation or denature condition of biotin-avidin bonds, the $3 \times$ FLAG tag may reduce contamination derived from nonspecific binding of affinity purification beads, for example. At least, the $3 \times$ FLAG and biotin-tagged CRISPR systems have been shown to achieve high yields of isolated interacting molecules.

\section{Technical Considerations}

\section{Design of gRNAs}

As mentioned earlier, the CRISPR complex can interfere with genomic functions and change chromatin accessibility when expressed in living cells. In addition, off-target binding of the CRISPR complexes can lead to nonspecific isolation of genomic regions. The design of target-specific gRNAs can overcome these issues and various design tools are available. ${ }^{43,44}$ Researchers can choose the appropriate gRNAs from the candidates listed from these design tools based on the following guidelines. First, to target promoter regions, gRNAs should be designed to bind several hundred base pairs upstream of the transcription start site to avoid interference with the recruitment of transcription factors and RNA polymerases by the CRISPR complex. In contrast, to target regulatory regions such as enhancers and silencers, the gRNA binding site should be located proximal to the regulatory sequence because they often have more distinct boundaries and binding of the CRISPR complex to the outside of those regulatory regions is less likely to interfere with their functions. Second, the target sites of gRNAs should not overlap with the binding sequences of endogenous DNAbinding molecules or sequences conserved among species, since these sequences may be functional regulatory regions. Third, on-target enrichment efficiency and specificity of gRNAs should be validated using CRISPR-based DNA purification combined with quantitative PCR (qPCR) and/or NGS. Finally, the genomic functions of target regions in the presence of the CRISPR complexes should be experimentally evaluated. If the gRNAs are not specific or interference with genomic functions is observed, the gRNAs or target sites should be reconsidered.

\section{Experimental controls}

One major concern of CRISPR-based DNA purification is the off-target binding of the CRISPR complex. To cancel out effects of the off-target binding and contamination of nonspecific molecules, proper control experiments should be conducted in parallel during CRISPR-based DNA purification. Experiments using cells lacking the gRNA target sequence provide a good reference for off-target binding and nonspecific interacting molecules. However, the deletion of the target genomic sequence is not always practical because it may require significant time and labor. Alternatively, the following comparison sets would be sufficient to exclude nonspecific binding molecules.

First, interacting molecules consistently detected with several different gRNAs for the same target genomic 
region are likely true positives because the off-target binding would differ among each gRNA. In parallel with this comparison set, the molecular profile obtained from cells expressing dCas9 alone or dCas9 plus an irrelevant gRNA would be helpful to exclude the contaminations of nonspecific binding molecules. Second, affinity purification using the same gRNA under different experimental conditions, for example, the presence or absence of stimulation and between different cell types, can identify the candidate molecules regulating the function of a genomic locus in a stimulation-dependent or cell typespecific manner. In the quantitative analysis, it is necessary to pay attention to the difference in efficiency of target enrichment between each condition. The difference in enrichment efficiency among different conditions is caused by various factors, such as chromatin accessibility and the expression levels of the CRISPR complex, leading to artifactual changes in the relative amounts of identified molecules. To avoid this problem, the observed quantitative changes can be normalized to the yield of a target region under each condition. In any case, however, additional independent analyses by other methods are required to unambiguously demonstrate the interaction of the candidate molecules with the target genomic region (details are discussed in the next section).

In the case of systems using enzymes biotinylating dCas9, nonspecific biotinylation of other proteins inside of cells may increase backgrounds. ${ }^{42}$ Therefore, additional controls such as samples expressing only the biotinylation enzyme must be required.

\section{Downstream Analyses of CRISPR-Based Purification of a Specific Locus \\ Identification of proteins associated \\ with a specific locus}

MS-based proteomics has been combined with CRISPRbased locus purification, enabling the comprehensive identification of interacting proteins. Many studies have applied the label-free "shotgun" MS approach to identify novel binding proteins in addition to known regulators of target regions. ${ }^{29,45-54}$ Quantitative proteomics using MS is more informative for characterizing the function of locus-specific binding proteins and their associated genomic regions. The relative amounts of associated proteins changing in response to extracellular stimulation can be detected by comparing the protein profile using stable isotope labeling by amino acids in cell culture (SILAC). ${ }^{55,56}$ It is postulated that the proteins that change in a stimulus-dependent manner may play essential roles in regulating gene function, that is, are candidates of regulator proteins. Compared with SILAC, the isobaric labeling strategies, such as tandem mass tags and isobaric tags for relative and absolute quantitation (iTRAQ), enable the simultaneous analysis of more samples, thereby increasing its throughput. The multiplex capacity of iTRAQ allows the sorting of locus-specific, off-target, and nonspecific binding proteins from a pool of proteins identified by CRISPR-based locus purification. This was achieved by comparison with multiple experimental controls, including CRISPR-based locus purification from cells lacking a gRNA target sequence, or cells expressing either dCas9 alone, or dCas9 with a gRNA targeting a different site. ${ }^{42}$

In addition to the detection of molecules associated with a specific locus, a potential application of CRISPRbased locus purification is the detection of novel posttranslational modifications (PTMs) of proteins, such as histones, transcription factors, and others. A vast diversity of PTMs and its combination on proteins contributes to gene transcription, DNA replication, and DNA repair. ${ }^{57,58}$ MS of cell extracts can identify and quantify the relative abundance of single and combinatorial PTMs in a large-scale manner. ${ }^{59}$ However, this analysis cannot detect the relatively low-abundant PTMs restricted in a specific genomic region and define its distribution. Enrichment of proteins at a specific genomic region by CRISPR-based locus purification can allow the detection of rare and locus-specific PTM combinations. Furthermore, their location information would help link the PTMs of proteins with genomic roles.

After detecting candidate proteins interacting with the locus of interest by CRISPR-based locus purification, further analysis is necessary to confirm the interaction and reveal functional relationships. In this respect, conventional ChIP is a powerful technique to detect the DNA-protein interactions in cells. Modern ChIP-based methods profile the genome-wide distribution of given DNA-binding proteins by combining with NGS or DNA microarray analysis. However, since the ChIP assay depends on the affinity and specificity of $\mathrm{Ab}$ in use, its application is restricted to the interaction with a known target protein; therefore, a stoichiometric comparison between different proteins is limited. Thus, the CRISPR-based locus purification and ChIP methods are complementary approaches to reveal unknown mechanisms underlying genomic events.

\section{Identification of RNAs associated}

with a specific locus

Accumulating evidence suggests that various types of RNA play essential roles in regulating genomic DNA functions such as gene transcription and genomic imprinting. ${ }^{60,61}$ Chromatin isolation by RNA purification (ChIRP) and capture hybridization analysis of RNA targets (CHART) are biochemical methods to identify 
chromatin-binding regions of an RNA of interest, similar to ChIP for proteins. ${ }^{62,63}$ Both methods are based on a hybridization-based strategy using biotin-tagged oligonucleotides to purify the target RNA and interacting chromatins. Similar to ChIP, these methods are based on the assumption that an RNA of interest interacts with the target genomic regions to be detected by PCR or other methods, unless nonbiased methods such as NGS are used.

Previously, a locus-specific DNA purification method using TAL has been combined with reverse transcriptionqPCR (RT-qPCR) or RNA sequencing (RNA-Seq) to identify RNAs associated with a specific genomic region. ${ }^{6,64}$ More recently, the CRISPR-based locus purification method combined with RNA-Seq has been used to detect various types of RNAs, including a circular RNA interacting with the FLII promoter and a micro-RNA interacting with the $I G F 2$ promoter. ${ }^{65,66}$ These methods are practical tools to reveal unknown RNA regulators of genomic functions.

\section{Identification of DNAs associated}

with a specific locus

Genomic DNA, compacted into nucleosomes, is further compartmentalized to organize the nuclear territories. This 3D genome architecture plays important roles in genomic functions. ${ }^{67}$ In particular, inter- or intrachromosomal regulatory elements, such as enhancers and silencers, interact with target genes by forming long-range DNA loops, resulting in distal gene regulation. The combined CRISPR-based locus purification and NGS analysis enables the identification of genomic regions associated with a specific locus without the need for a ligation step. In this approach, a target locus is tagged in-cell or in vitro with a CRISPR complex, affinity purified, followed by subsequent NGS analysis to identify the interacting regions. ${ }^{12,68}$ This analysis results in the detection of many interacting genomic regions, which are candidates of regulatory elements of a target gene. Additional experimental controls and analyses are required to narrow down these candidates. These include negative control cells to compare the interacting regions, characterization of epigenetic markers, analysis of open chromatin structures detected with formaldehyde-associated isolation of regulatory elements (FAIRE) or assay for transposase-accessible chromatin with high-throughput sequencing (ATAC-Seq), and deletion of candidate genomic regions. ${ }^{69,70}$ For example, the combined approach of CRISPR-based locus purification and NGS analysis identified enhancer regions overlapping with open chromatin regions and epigenetic markers of $\mathrm{H} 3 \mathrm{~K} 4 \mathrm{mel}$ and H3K27Ac. Moreover, the deletion of these accessible regions resulted in the downregulation of target genes. ${ }^{12,49,71}$
Together, these studies have demonstrated the capability of the CRISPR-based approach for identification of locus-specific interactions between a promoter and an enhancer(s).

The chromosome conformation capture (3C), and its derivative methods, have advanced the understanding of genome interaction and chromatin architecture. ${ }^{72-76}$ The basic concept behind the 3C-based methods is that the frequency of ligation correlates with the physical DNA-DNA interactions. However, because this is an indirect detection approach, there is a potential risk of detecting not only physical interactions but also accessible genomic regions, thereby occasionally leading to discrepancies with other techniques. ${ }^{77,78}$ Several methods have been developed to detect the physical genome interactions in a ligation-free manner, including genome architecture mapping (GAM), split-pool recognition of interactions by tag extension (SPRITE), and chromatin interaction analysis via dropletbased and barcode-linked sequencing (ChIA-Drop). ${ }^{79-81}$ GAM measures the statistical proximities of chromosomes by sequencing the DNA extracted from ultrathin cryosectioned nuclear slices. SPRITE includes repeated rounds of splitting and barcoding of individual chromatin complexes, and subsequent identification of the interacting genomic regions by matching the terminal barcodes. In the case of ChIA-Drop, amplicons arising from gel-bead-inemulsion (GEM) droplets of each chromatin complex are tracked with a barcode sequence. These methods allow the genome-wide mapping of simultaneous contacts of chromatin; in contrast, the CRISPR-based locus purification approach detects multiple genomic regions interacting with one target locus. Advantageously, it does not require specialized equipment nor training to perform because it is a similar procedure to ChIP-Seq after tagging the target locus with the CRISPR complex. In summary, the combination of CRISPR-based locus purification and NGS is a practical and cost-effective approach to identify regulatory elements interacting with a genomic region of interest.

\section{Concluding Remarks}

There is a rising consensus that macromolecules consisting of numerous proteins, RNAs, and DNAs orchestrate the series of nuclear events such as the chromatin organization and gene transcription. To help define the regulatory principles, we need to consider both the role of single molecule and the global control of genomic functions. Purification of a specific genomic locus was previously hindered by technological limitations; however, the advances of engineered DNA-binding molecules have provided a feasible and flexible approach. In this study, we described the recent advances of CRISPR-based DNA purification tools. The CRISPR-based DNA purification 
system, coupled with a high-throughput identification method, can allow a comprehensive identification of molecules interacting with a specific locus to provide snapshots of the genomic process. This approach will provide new insights into how the function of a specific locus is regulated. Therefore, the CRISPR-based DNA purification approach will facilitate the unbiased discovery of chromosome biology.

\section{Authors' Contributions}

All authors participated in the writing and editing of the article.

\section{Author Disclosure Statement}

H. Fujii and T.F. are inventors of granted patents owned by Osaka University for the technology of purification and subsequent analysis of specific DNA including genomic DNA with chromatin structure using insertion of a recognition sequence of an exogenous DNA-binding molecule such as LexA into the target DNA and affinity purification of the exogenous DNA-binding molecule bound to its recognition sequence (Patent name: "Method for isolating specific genomic regions", Patent numbers: US 8,415,098, Japan 5,413,924). Osaka University licensed the patents to Epigeneron, Inc., H. Fujii and T.F. are also inventors of granted patents and a patent pending owned by Osaka University for the technology of purification and subsequent analysis of specific DNA including genomic DNA with chromatin structure using an engineered DNA-binding molecule including the CRISPR complex binding to the target DNA (Patent name: "Method for isolating specific genomic region using molecule binding specifically to endogenous DNA sequence," Patent numbers: Japan 5,954,808, EP 2,963,113; Patent application number: WO2014/ 125668). Osaka University licensed the patents to Active Motif, Inc., and Epigeneron, Inc., H. Fujii and T.F. are co-founders of Epigeneron, Inc. H. Fujii and T.F. are a director and an advisor of Epigeneron, Inc., respectively. $\mathrm{H}$. Fujii is a member of the advisory board of Addgene. H. Fujita has no conflicts of interest with the contents of this study.

\section{Funding Information}

This study was supported by the Karoji Memorial Fund for Medical Research (H. Fujii), Grant-in-Aid for Scientific Research (C) (No. 18K06176) (T.F.), and Grant-inAid for Scientific Research (B) (No. 15H04329) (T.F., H. Fujii), "Transcription Cycle" (No. 15H01354) (H. Fujii) from the Ministry of Education, Culture, Sports, Science and Technology of Japan.

\section{References}

1. Li B, Carey M, Workman JL. The role of chromatin during transcription. Cell. 2007;128:707-719. DOI: 10.1016/j.cell.2007.01.015.

2. Zentner GE, Henikoff S. Regulation of nucleosome dynamics by histone modifications. Nat Struct Mol Biol. 2013;20:259-266. DOI: 10.1038/ nsmb.2470.

3. Kim T-K, Shiekhattar R. Architectural and functional commonalities between enhancers and promoters. Cell. 2015;162:948-959. DOI: 10.1016/ j.cell.2015.08.008.

4. Hoshino A, Fujii H. Insertional chromatin immunoprecipitation: A method for isolating specific genomic regions. J Biosci Bioeng. 2009;108:446449. DOI: 10.1016/j.jbiosc.2009.05.005.

5. McCullagh E, Seshan A, El-Samad H, et al. Coordinate control of gene expression noise and interchromosomal interactions in a MAP kinase pathway. Nat Cell Biol. 2010;12:954-962. DOI: 10.1038/ncb2097.

6. Fujita T, Fujii H. Direct identification of insulator components by insertional chromatin immunoprecipitation. PLoS One. 2011;6:e26109. DOI: 10.1371/journal.pone.0026109.

7. Fujita T, Fujii H. Efficient isolation of specific genomic regions by insertional chromatin immunoprecipitation (iChIP) with a secondgeneration tagged LexA DNA-binding domain. Adv Biosci Biotechnol. 2012;3:626-629. DOI: 10.4236/abb.2012.35081.

8. Agelopoulos M, McKay DJ, Mann RS. Developmental regulation of chromatin conformation by Hox proteins in Drosophila. Cell Rep. 2012;1:350-359. DOI: 10.1016/j.celrep.2012.03.003.

9. Byrum SD, Raman A, Taverna SD, et al. ChAP-MS: A method for identification of proteins and histone posttranslational modifications at a single genomic locus. Cell Rep. 2012;2:198-205. DOI: 10.1016/ j.celrep.2012.06.019.

10. Pourfarzad F, Aghajanirefah A, de Boer E, et al. Locus-specific proteomics by TChP: Targeted chromatin purification. Cell Rep. 2013;4:589-600. DOI: 10.1016/j.celrep.2013.07.004.

11. Fujita T, Kitaura F, Fujii H. A critical role of the Thy28-MYH9 axis in B cellspecific expression of the Pax5 gene in Chicken B cells. PLoS One. 2015;10:e0116579. DOI: 10.1371/journal.pone.0116579.

12. Fujita T, Kitaura F, Yuno M, et al. Locus-specific ChIP combined with NGS analysis reveals genomic regulatory regions that physically interact with the Pax5 promoter in a chicken B cell line. DNA Res. 2017;24:537548. DOI: 10.1093/dnares/ds $\times 023$.

13. Bui PL, Nishimura K, Seminario Mondejar G, et al. Template activating factor-I $\alpha$ regulates retroviral silencing during reprogramming. Cell Rep. 2019;29:1909-1922.e5. DOI: 10.1016/j.celrep.2019.10.010.

14. Husain A, Xu J, Fujii $H$, et al. SAMHD1-mediated dNTP degradation is required for efficient DNA repair during antibody class switch recombination. EMBO J. 2020;n/a:e102931. DOI: 10.15252/embj.2019102931.

15. Fujita $\mathrm{T}$, Fujii $\mathrm{H}$. Efficient isolation of specific genomic regions retaining molecular interactions by the iChIP system using recombinant exogenous DNA-binding proteins. BMC Mol Biol. 2014;15:26. DOI: 10.1186/ s12867-014-0026-0.

16. Déjardin J, Kingston RE. Purification of proteins associated with specific genomic Loci. Cell. 2009;136:175-186. DOI: 10.1016/ j.cell.2008.11.045.

17. Saksouk N, Barth TK, Ziegler-Birling C, et al. Redundant mechanisms to form silent chromatin at pericentromeric regions rely on BEND3 and DNA methylation. Mol Cell. 2014;56:580-594. DOI: 10.1016/j.molcel.2014.10.001.

18. Zeng Z, Jiang J. Isolation and proteomics analysis of barley centromeric chromatin using PICh. J Proteome Res. 2016;15:1875-1882. DOI: 10.1021/acs.jproteome.6b00063.

19. Ide S, Dejardin J. End-targeting proteomics of isolated chromatin segments of a mammalian ribosomal RNA gene promoter. Nat Commun. 2015;6:6674. DOI: 10.1038/ncomms7674.

20. Kim YG, Cha J, Chandrasegaran S. Hybrid restriction enzymes: Zinc finger fusions to Fok I cleavage domain. Proc Natl Acad Sci U S A. 1996;93:1156-1160. DOI: 10.1073/pnas.93.3.1156.

21. Boch J, Scholze $H$, Schornack S, et al. Breaking the code of DNA binding specificity of TAL-type III effectors. Science. 2009;326:1509-1512. DOI: 10.1126/science.1178811.

22. Christian M, Cermak T, Doyle EL, et al. Targeting DNA double-strand breaks with TAL effector nucleases. Genetics. 2010;186:757-761. DOI: 10.1534/genetics.110.120717. 
23. Urnov FD, Rebar EJ, Holmes MC, et al. Genome editing with engineered zinc finger nucleases. Nat Rev Genet. 2010;11:636-646. DOI: 10.1038/ nrg2842.

24. Jinek M, Chylinski K, Fonfara I, et al. A programmable dual-RNA-guided DNA endonuclease in adaptive bacterial immunity. Science. 2012;337:816-821. DOI: 10.1126/science.1225829.

25. Gasiunas G, Barrangou R, Horvath $P$, et al. Cas9-crRNA ribonucleoprotein complex mediates specific DNA cleavage for adaptive immunity in bacteria. Proc Natl Acad Sci U S A. 2012;109:E2579-E2586. DOI: 10.1073/ pnas.1208507109.

26. Qi LS, Larson MH, Gilbert LA, et al. Repurposing CRISPR as an RNA-guided platform for sequence-specific control of gene expression. Cell. 2013;152:1173-1183. DOI: 10.1016/j.cell.2013.02.022.

27. Xu X, Qi LS. A CRISPR-dCas toolbox for genetic engineering and synthetic biology. J Mol Biol. 2019;431:34-47. DOI: 10.1016/j.jmb.2018.06.037.

28. Pickar-Oliver A, Gersbach CA. The next generation of CRISPR-Cas technologies and applications. Nat Rev Mol Cell Biol. 2019;20:490-507. DOI: 10.1038/s41580-019-0131-5.

29. Fujita T, Fujii H. Efficient isolation of specific genomic regions and identification of associated proteins by engineered DNA-binding molecule-mediated chromatin immunoprecipitation (enChIP) using CRISPR. Biochem Biophys Res Commun. 2013;439:132-136. DOI: 10.1016/ j.bbrc.2013.08.013.

30. Fujita $T$, Yuno $M$, Fujii $H$. Efficient sequence-specific isolation of DNA fragments and chromatin by in vitro enChIP technology using recombinant CRISPR ribonucleoproteins. Genes Cells. 2016;21:370-377. DOI: 10.1111/gtc.12341.

31. Gammage PA, Moraes CT, Minczuk M. Mitochondrial genome engineering: The revolution may not be CRISPR-Ized. Trends Genet. 2018;34:101110. DOI: 10.1016/j.tig.2017.11.001.

32. Yoo B-C, Yadav NS, Orozco EM, et al. Cas9/gRNA-mediated genome editing of yeast mitochondria and Chlamydomonas chloroplasts. PeerJ. 2020;8:e8362. DOI: 10.7717/peerj.8362.

33. Polstein LR, Perez-Pinera P, Kocak DD, et al. Genome-wide specificity of DNA binding, gene regulation, and chromatin remodeling by TALE- and CRISPR/Cas9-based transcriptional activators. Genome Res. 2015;25:1158-1169. DOI: 10.1101/gr.179044.114.

34. Barkal AA, Srinivasan $S$, Hashimoto $T$, et al. Cas9 functionally opens chromatin. PLoS One. 2016;11:e0152683. DOI: 10.1371/journal.pone.0152683.

35. Hardenbol $P$, Banér J, Jain $M$, et al. Multiplexed genotyping with sequence-tagged molecular inversion probes. Nat Biotechnol. 2003;21:673-678. DOI: 10.1038/nbt821.

36. Albert TJ, Molla MN, Muzny DM, et al. Direct selection of human genomic loci by microarray hybridization. Nat Methods. 2007;4:903-905. DOI: 10.1038/nmeth1111.

37. Hayden MJ, Nguyen TM, Waterman A, et al. Multiplex-ready PCR: A new method for multiplexed SSR and SNP genotyping. BMC Genomics. 2008;9:80. DOI: 10.1186/1471-2164-9-80.

38. Ng SB, Turner EH, Robertson PD, et al. Targeted capture and massively parallel sequencing of 12 human exomes. Nature. 2009;461:272-276. DOI: 10.1038 /nature08250.

39. Lee J, Lim H, Jang H, et al. CRISPR-Cap: Multiplexed double-stranded DNA enrichment based on the CRISPR system. Nucleic Acids Res. 2019;47:e1. DOI: $10.1093 /$ nar/gky820.

40. Slesarev A, Viswanathan L, Tang Y, et al. CRISPR/CAS9 targeted CAPTURE of mammalian genomic regions for characterization by NGS. Sci Rep. 2019;9:3587. DOI: 10.1038/s41598-019-39667-4.

41. Amarasinghe SL, Su S, Dong X, et al. Opportunities and challenges in long-read sequencing data analysis. Genome Biol. 2020;21:30. DOI: 10.1186/s13059-020-1935-5.

42. Liu X, Zhang Y, Chen $Y$, et al. In situ capture of chromatin interactions by biotinylated dCas9. Cell. 2017;170:1028-1043.e19. DOI: 10.1016/ j.cell.2017.08.003.

43. Cui $Y, X u$ J, Cheng M, et al. Review of CRISPR/Cas9 sgRNA design tools. Interdiscip Sci. 2018;10:455-465. DOI: 10.1007/s12539-018-0298-z.

44. Sledzinski P, Nowaczyk M, Olejniczak M. Computational tools and resources supporting CRISPR-Cas experiments. Cells. 2020;9:1288. DOI: 10.3390/cells9051288.

45. Fujita T, Asano Y, Ohtsuka J, et al. Identification of telomere-associated molecules by engineered DNA-binding molecule-mediated chromatin immunoprecipitation (enChIP). Sci Rep. 2013;3:3171. DOI: 10.1038/ srep03171.
46. Tsui C, Inouye C, Levy M, et al. dCas9-targeted locus-specific protein isolation method identifies histone gene regulators. Proc Natl Acad Sci U S A. 2018;115:E2734-E2741. DOI: 10.1073/pnas.1718844115.

47. Shamsi F, Xue R, Huang TL, et al. FGF6 and FGF9 regulate UCP1 expression independent of brown adipogenesis. Nat Commun. 2020;11:1421. DOI: 10.1038/s41467-020-15055-9.

48. Waldrip ZJ, Byrum SD, Storey AJ, et al. A CRISPR-based approach for proteomic analysis of a single genomic locus. Epigenetics. 2014;9:1207-1211. DOI: 10.4161/epi.29919.

49. Mochizuki Y, Chiba T, Kataoka K, et al. Combinatorial CRISPR/Cas9 approach to elucidate a far-upstream enhancer complex for tissuespecific Sox9 expression. Dev Cell. 2018;46:794-806.e6. DOI: 10.1016/ j.devcel.2018.07.024.

50. Fang F, Xia N, Angulo B, et al. A distinct isoform of ZNF207 controls self-renewal and pluripotency of human embryonic stem cells. Nat Commun. 2018;9:4384. DOI: 10.1038/s41467-018-06908-5.

51. Han B, Zhou B, Qu Y, et al. FOXC1-induced non-canonical WNT5A-MMP7 signaling regulates invasiveness in triple-negative breast cancer. Oncogene. 2018;37:1399-1408. DOI: 10.1038/s41388-017-0021-2.

52. Campbell AE, Shadle SC, Jagannathan $S$, et al. NuRD and CAF-1mediated silencing of the D4Z4 array is modulated by DUX4-induced MBD3L proteins. Heard E, ed. eLife. 2018;7:e31023. DOI: 10.7554/ eLife.31023.

53. González-Rico FJ, Vicente-García C, Fernández A, et al. Alu retrotransposons modulate Nanog expression through dynamic changes in regional chromatin conformation via aryl hydrocarbon receptor. Epigenetics Chromatin. 2020;13:15. DOI: 10.1186/s13072-020-00336-w.

54. Huang $Y$, Xiang $Y$, Xie Z, et al. Mass spectrometry-based proteomic capture of proteins bound to the MACC1 promoter in colon cancer. Clin Exp Metastasis. 2020;37:477-487. DOI: 10.1007/s10585-020-10045-z.

55. Fujita $\mathrm{T}$, Fujii $\mathrm{H}$. Identification of proteins associated with an IFN $\gamma$ responsive promoter by a retroviral expression system for enChIP using CRISPR. PLoS One. 2014;9:e103084. DOI: 10.1371/journal.pone.0103084.

56. Hamidian A, Vaapil M, von Stedingk K, et al. Promoter-associated proteins of EPAS1 identified by enChIP-MS-A putative role of HDX as a negative regulator. Biochem Biophys Res Commun. 2018;499:291-298. DOI: 10.1016/j.bbrc.2018.03.150.

57. Filtz TM, Vogel WK, Leid M. Regulation of transcription factor activity by interconnected post-translational modifications. Trends Pharmacol Sci. 2014;35:76-85. DOI: 10.1016/j.tips.2013.11.005.

58. Chan JC, Maze I. Nothing is yet set in (Hi)stone: Novel post-translational modifications regulating chromatin function. Trends Biochem Sci. 2020;45:829-844. DOI: 10.1016/j.tibs.2020.05.009.

59. Yuan Z-F, Arnaudo AM, Garcia BA. Mass spectrometric analysis of histone proteoforms. Annu Rev Anal Chem (Palo Alto Calif). 2014;7:113-128. DOI: 10.1146/annurev-anchem-071213-015959.

60. Lee JT, Bartolomei MS. X-inactivation, imprinting, and long noncoding RNAs in health and disease. Cell. 2013;152:1308-1323. DOI: 10.1016/ j.cell.2013.02.016.

61. Arnold PR, Wells AD, Li XC. Diversity and emerging roles of enhancer RNA in regulation of gene expression and cell fate. Front Cell Dev Biol. 2019;7:377. DOI: 10.3389/fcell.2019.00377.

62. Chu C, Qu K, Zhong FL, et al. Genomic maps of long noncoding RNA occupancy reveal principles of RNA-chromatin interactions. Mol Cell. 2011;44:667-678. DOI: 10.1016/j.molcel.2011.08.027.

63. Simon MD, Wang $\mathrm{Cl}$, Kharchenko PV, et al. The genomic binding sites of a noncoding RNA. Proc Natl Acad Sci U S A. 2011;108:20497-20502. DOI: 10.1073/pnas.1113536108.

64. Fujita T, Yuno M, Okuzaki D, et al. Identification of non-coding RNAs associated with telomeres using a combination of enChIP and RNA sequencing. PLoS One. 2015;10:e0123387. DOI: 10.1371/ journal.pone.0123387.

65. Zhang $\mathrm{Y}, \mathrm{Hu}$ J-F, Wang $\mathrm{H}$, et al. CRISPR Cas9-guided chromatin immunoprecipitation identifies miR483 as an epigenetic modulator of IGF2 imprinting in tumors. Oncotarget. 2017;8:34177-34190. DOI: 10.18632/ oncotarget.10918.

66. Chen N, Zhao G, Yan X, et al. A novel FLI1 exonic circular RNA promotes metastasis in breast cancer by coordinately regulating TET1 and DNMT1. Genome Biol. 2018;19:218. DOI: 10.1186/s13059-018-1594-y.

67. Maass PG, Barutcu AR, Rinn JL. Interchromosomal interactions: A genomic love story of kissing chromosomes. J Cell Biol. 2019;218:27-38. DOI: 10.1083/jcb.201806052. 
68. Fujita T, Yuno M, Suzuki Y, et al. Identification of physical interactions between genomic regions by enChIP-Seq. Genes Cells. 2017;22:506520. DOI: $10.1111 /$ gtc. 12492 .

69. Giresi PG, Kim J, McDaniell RM, et al. FAIRE (Formaldehyde-Assisted Isolation of Regulatory Elements) isolates active regulatory elements from human chromatin. Genome Res. 2007;17:877-885. DOI: 10.1101/ gr.5533506.

70. Buenrostro JD, Giresi PG, Zaba LC, et al. Transposition of native chromatin for fast and sensitive epigenomic profiling of open chromatin, DNAbinding proteins and nucleosome position. Nat Methods. 2013;10:1213-1218. DOI: 10.1038/nmeth.2688.

71. Seo W, Shimizu K, Kojo S, et al. Runx-mediated regulation of CCL5 via antagonizing two enhancers influences immune cell function and antitumor immunity. Nat Commun. 2020;11:1562. DOI: 10.1038/s41467020-15375-w.

72. Dekker J, Rippe K, Dekker M, et al. Capturing chromosome conformation. Science. 2002;295:1306-1311. DOI: 10.1126/science.1067799.

73. Dostie J, Richmond TA, Arnaout RA, et al. Chromosome Conformation Capture Carbon Copy (5C): A massively parallel solution for mapping interactions between genomic elements. Genome Res. 2006;16:12991309. DOI: $10.1101 /$ gr.5571506.

74. Simonis $M$, Klous $P$, Splinter $E$, et al. Nuclear organization of active and inactive chromatin domains uncovered by chromosome conformation capture-on-chip (4C). Nat Genet. 2006;38:1348-1354. DOI: 10.1038/ng1896.

75. Zhao Z, Tavoosidana G, Sjölinder M, et al. Circular chromosome conformation capture (4C) uncovers extensive networks of epigenetically regulated intra- and interchromosomal interactions. Nat Genet. 2006;38:1341-1347. DOI: 10.1038/ng1891.

76. Lieberman-Aiden $\mathrm{E}$, van Berkum NL, Williams L, et al. Comprehensive mapping of long-range interactions reveals folding principles of the human genome. Science. 2009;326:289-293. DOI: 10.1126/science.1181369.

77. Williamson I, Berlivet S, Eskeland R, et al. Spatial genome organization: Contrasting views from chromosome conformation capture and fluorescence in situ hybridization. Genes Dev. 2014;28:2778-2791. DOI: 10.1101/gad.251694.114.

78. Finn EH, Pegoraro G, Brandão HB, et al. Extensive heterogeneity and intrinsic variation in spatial genome organization. Cell. 2019;176:15021515.e10. DOI: 10.1016/j.cell.2019.01.020.
79. Beagrie RA, Scialdone A, Schueler M, et al. Complex multi-enhancer contacts captured by genome architecture mapping. Nature. 2017;543:519-524. DOI: 10.1038/nature21411.

80. Quinodoz SA, Ollikainen N, Tabak B, et al. Higher-order interchromosomal hubs shape 3D genome organization in the nucleus. Cell. 2018;174:744-757.e24. DOI: 10.1016/j.cell.2018.05.024.

81. Zheng M, Tian SZ, Capurso D, et al. Multiplex chromatin interactions with single-molecule precision. Nature. 2019;566:558-562. DOI: 10.1038/ s41586-019-0949-1.

82. Fujita T, Yuno M, Fujii H. enChIP systems using different CRISPR orthologues and epitope tags. BMC Res Notes. 2018;11:154. DOI: 10.1186/ s13104-018-3262-4.

83. Fujita T, Yuno M, Fujii H. Allele-specific locus binding and genome editing by CRISPR at the p16INK4a locus. Sci Rep. 2016;6:30485. DOI: 10.1038/ srep30485.

84. Fujita T, Kitaura F, Oji A, et al. Transgenic mouse lines expressing the $3 \times$ FLAG-dCas9 protein for enChIP analysis. Genes Cells. 2018;23:318325. DOI: $10.1111 /$ gtc. 12573 .

85. Fujita T, Yuno M, Fujii H. An enChIP system for the analysis of bacterial genome functions. BMC Res Notes. 2018;11:387. DOI: 10.1186/s13104018-3486-3.

86. Kuscu C, Mammadov R, Czikora A, et al. Temporal and spatial epigenome editing allows precise gene regulation in mammalian cells. $J \mathrm{Mol} \mathrm{Biol}$. 2019;431:111-121. DOI: 10.1016/j.jmb.2018.08.001.

87. Baumgarten S, Bryant JM, Sinha A, et al. Transcriptome-wide dynamics of extensive m6A mRNA methylation during Plasmodium falciparum blood-stage development. Nat Microbiol. 2019;4:2246-2259. DOI: 10.1038/s41564-019-0521-7.

88. Carpenter MD, Hu Q, Bond AM, et al. Nr4a1 suppresses cocaine-induced behavior via epigenetic regulation of homeostatic target genes. Nat Commun. 2020;11:504. DOI: 10.1038/s41467-020-14331-y.

89. Kuhl L-M, Makrantoni V, Recknagel S, et al. A dCas9-based system identifies a central role for Ctf19 in kinetochore-derived suppression of meiotic recombination. Genetics. 2020;216:395-408. DOI: 10.1534/genetics.120.303384.

90. Bryant JM, Baumgarten S, Dingli F, et al. Exploring the virulence gene interactome with CRISPR/dCas9 in the human malaria parasite. Mol Syst Biol. 2020;16:e9569. DOI: 10.15252/msb.20209569. 